\title{
微处理器敏捷设计方法综述
}

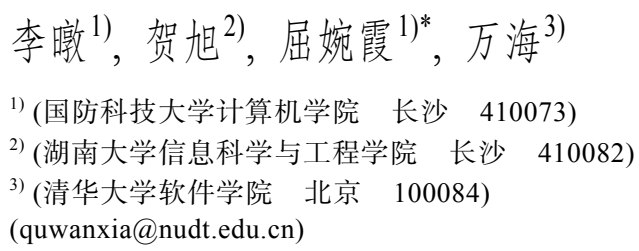

\begin{abstract}
摘 要: 随着登纳德缩放定律和摩尔定律几近终结, 通过领域特定体系结构提升微处理器性能变得越来越重要, 迫 切需要提升微处理器设计生产率来应对网络、智能、安全等领域特定需求. 国内外的实践表明, 微处理器敏捷设计方 法是一种能有效提升微处理器设计生产率的方法. 通过对比软硬件设计的差异, 分析出敏捷设计的本质及其应用于 微处理器设计所面临的挑战. 综述了微处理器敏捷设计领域近年来代表性研究实践, 归纳出微处理器敏捷设计关键 使能技术，探讨了该领域未来潜在的研究方向.
\end{abstract}

关键词：敏捷设计；微处理器; 领域特定语言; 电子设计自动化

中图法分类号: TP391.41 DOI: 10.3724/SP.J.1089.2021.18229

\section{Survey on Microprocessor Agile Design Methodology and Technology}

\author{
Li Tun ${ }^{1)}, \mathrm{He} \mathrm{Xu}^{2}$, Qu Wanxia ${ }^{1)^{*}}$, and Wan $\mathrm{Hai}^{3)}$ \\ 1) (College of Computer Science and Technology, National University of Defense Technology, Changsha 410073) \\ 2) (College of Computer Science and Electronic Engineering, Hunan University, Changsha 410082) \\ 3) (School of Software, Tsinghua University, Beijing 100084)
}

\begin{abstract}
The era of Dennard scaling and Moore's law is coming to an end. As a result, it is more and more important to improve the performance of microprocessors through novel domain specific architectures. The growing complexity of domain specific architectures leads to a design productivity crisis, which stimulates the development of new tools and methodologies to enable the completion of complex chip designs on schedule and within budget for specific domain requirements. The global research practices gradually show that the microprocessor agile design methodology is an effective solution to the design productivity crisis. This survey first briefly discusses the essence of agile design methodology and the possibility of applying it to microprocessor design by comparing the characters between software and hardware designs. Then the paper reviews the main research work in recent years and shows the research status and development trends. Finally, we give some suggestions on the potential research directions for the near future.
\end{abstract}

Key words: agile design; microprocessor; domain specific language; electronic design automation

随着信息技术应用越来越广，需要更多具备 新功能的应用软件来满足各类需求. 而随着集成
电路技术发展，工艺尺寸不断缩小，进人后摩尔时 代后, 登纳德缩放定律和摩尔定律几近终结, 意味

收稿日期: 2020-02-09; 修回日期: 2020-07-06. 基金项目: 国家自然科学基金(U19A2062); 国家重点研发计划(2018YFB0204301). 李暾(1974-), 男, 博士, 教授, 博士生导师, CCF 高级会员, 主要研究方向为电子设计自动化; 贺旭(1985一), 女, 博士, 副教授, 硕 士生导师, 主要研究方向为人工智能、物理设计 CAD; 屈婉霞(1972-), 女, 博士, 副研究员, 论文通讯作者, 主要研究方向为体系结 构、处理器设计; 万海(1981一), 男, 博士, 副研究员, 硕士生导师, 主要研究方向为实时以太网、形式化建模与验证. 
着晶体管性能的快速提升将急剧变缓，尤其是试 图通过工艺尺寸的缩减来提高微处理器的性能、功 耗等指标, 将变得越来越困难 ${ }^{[1]}$. 此外, 通用微处 理器固有的低效率，越来越难以通过提升其性能 来满足新软件功能在性能、功耗等方面的需求 ${ }^{[2]}$. 因此，国内外学术界和工业界越来越多地通过专 用芯片 (application specific integrated circuit, ASIC) 来满足应用的各种质量需求. 这也表明研究微处 理器设计和架构的新方法变得越来越重要, 需要 新的架构和开发方法来快速生成针对不同市场的 高效专用芯片。

为应对不同市场需求，常常需要从功能需求、 体系结构开始，设计新的领域专用芯片，集成更多 功能. 虽然 ASIC 设计会带来更好的性能和更低的 功耗，但是，首先 ASIC 的设计成本非常高; 其次, 这类芯片在初始应用时，其流片量一般不会太大. 据美国国防部高级研究计划局(defense advanced research projects agency, DARPA)统计，美国国防 部对芯片的需求特点是流片量在 100 万片以下. 该 情况下，芯片设计开销将超过流片开销，占到芯片 总成本的 $69 \% \sim 92 \%{ }^{[3]}$. 此时的制约因素主要有 18 24 个月的设计周期、更多的设计验证投人、缺 乏可重用的 IP 等.

缩短设计周期、降低设计成本和提高设计生产 率, 已成为微处理器设计面临的一个重要挑战. 从 2010 年起, 在微处理器设计领域, 学术界和业界 一直在寻求提升 ASIC 设计生产率的方法, 核心理 念是对现有微处理器设计方法进行变革, 通过设 计方法、设计工具链的革新, 使得微处理器设计流 程变“轻”, 以提升设计生产率.

微处理器敏捷设计方法正是这一理念的产物. 该方法借鉴软件设计方法，引人敏捷设计的理念， 本质是要提高微处理器设计生产率. 近几年来, 敏 捷硬件设计方法已成为国内外微处理器设计领域 的研究热点之一，一些成功案例显示了其强大的 设计效能. 例如, 加州大学伯克利分校以学生为主 的十几人团队，应用敏捷设计方法，5 年内成功流 片 12 次 ${ }^{[4]}$. 斯坦福大学类似结构的研究团队, 2 年 内成功流片 2 次 ${ }^{[5]}$.

近年来, 微处理器敏捷设计的研究越来越受 到重视. 在国家层面, 2018 年美国 DARPA 启动了
电子复兴计划 ${ }^{\circledR}$, 通过快速电路实现(circuit realization at faster timescales, CRAFT) 项目资助了一系 列高校、研究机构和企业开展相关研究, 代表性高 校有加州大学伯克利分校、麻省理工学院、哈佛大 学和斯坦福大学等高校, 代表性企业有电子设计 自动化工具(electronic design automation, EDA)巨 头 Cadence 和 Synopsis 公司. 在校企合作层面, 2018 年, Intel 成立了敏捷硬件设计科技中心(Intel's science and technology center for agile hardware design, ISTC Agile), 分别在加州大学伯克利分校 启动了 Aspire 项目 ${ }^{(2)}$, 在斯坦福大学成立了 AHA 敏捷硬件中心(AHA! agile hardware center), 启动 了 AHA 敏捷硬件项目(AHA! agile hard- ware project, AHA $)^{3}$. 其中, Aspire 项目分别选取嵌人式可 编程视觉处理器和 Berkeley 仓库级计算机 (warehouse-scale computers, WSC)芯片 FireBox 作 为敏捷设计的应用场景，而 $\mathrm{AHA}$ 项目选取使用图 像/视觉计算作为其应用领域.

本文将所有为提高微处理器设计生产率的研 究都归于敏捷设计相关研究，基于该分类，对国内 外到目前为止的微处理器敏捷设计相关研究进行 综述, 包括敏捷设计的本质、微处理器敏捷设计代 表性工作、微处理器敏捷设计关键使能技术，最后， 列出了未来潜在的研究方向.

\section{1 敏捷设计的本质}

敏捷的本质是迅速、快捷，即迅速响应需求， 快速反馈结果. 传统的产品设计方法是一种瀑布模 型, 一个产品通常会经历市场发现、产品定义、产 品开发、产品发布等阶段, 它强调设计流程的循序 渐进性. 而随着市场竞争的加剧, 对缩短产品上市 时间的需求越来越高，瀑布模型固有的线性开发流 程、固定开发阶段划分以及难以适应用户需求变化 等缺点, 导致其开发耗时较长, 产品投人市场后的 失败风险大大提升，难以适应新的产品开发需求。

敏捷设计强调高效并且持续不断地进行设计 以达到客户满意的标准，即交付的产品是逐步递 增的，而不必等到一个设计阶段完全结束后才开始 下一阶段. 所以敏捷设计是一种可持续化的设计流 程, 每一次迭代都将快速经历产品开发的各阶段.

\footnotetext{
(1) https://www.darpa.mil/work-with-us/electronics-resurgence-initiative

(2) https://aspire.eecs.berkeley.edu/agile-hw-design-center

(3) https://aha.stanford.edu
} 
每次迭代只增加一部分功能和特征, 频繁的测试 与修改是敏捷设计的首要原则. 敏捷设计方法也 强调开发团队与客户的紧密联系, 能及时发现真 正的需求痛点. 综上, 敏捷设计是一种理念, 各领 域的产品开发都可对其进行适配.

在信息科学领域，敏捷设计最早应用于软件 开发, 提出了敏捷软件开发 12 原则 ${ }^{[6]}$. 敏捷软件 开发方法作为一种轻量级开发方法, 强调开发过 程中用户的参与，使得开发的软件可以和用户的 需求一同发展. 自敏捷设计方法引人软件开发领 域以来, 各类数据表明, 在投放市场后, 使用敏捷 设计模式的项目成功率要远远高于传统设计模式 下的产物.

传统共识中微处理器设计的需求非常稳定, 不存在不断创建产品交付客户这样需要不断迭代 的循环过程, 即不太可能应用敏捷设计方法. 但 是，进人后摩尔时代后，登纳德缩放定律和摩尔定 律走向终结, 微处理器设计和架构的新方法变得 越来越重要, 需要领域特定体系结构(domain specific architecture, DSA)和新的开发方法, 来快速生 成针对不同应用需求的高效专用芯片 ${ }^{[2]}$. 因此, 集 成电路设计领域也将面临应用需求不断变化、快速 应对应用需求和芯片快速上市等压力. 芯片设计 方法对提高微处理器设计效率的重要性越来越高. 与软件开发领域相比, 将敏捷设计方法应用于微 处理器设计已成为一种可选的解决方法.

将敏捷设计方法成功应用于芯片设计, 需将 芯片设计与软件设计进行对比, 寻求合适的切人 点，以及确定在多大范围内能应用敏捷设计方法. 本文认为, 二者在以下几个方面存在本质区别:

(1) 软件开发过程中规约文档较为灵活, 往往 会带来模糊性，而芯片设计中，标准化规约通常更 具有结构性、规则性.

(2) 生成软件所需的工具较为单纯，基本上只 需要一个编译器, 而生成芯片需要依赖大量的 EDA 工具，涉及逻辑、电子、物理等领域的知识.

(3) 软件开发的产品是相对“软”的程序代码, 易于修改. 而芯片设计因存在物理设计环节，越接 近设计的后端，编码越“硬”，设计越难以修改.

（4）软件开发流程虽是瀑布模型，但模型中层 次的概念较弱. 而芯片开发中, 设计层次非常重要, 一个设计将经历系统层、寄存器传输层(register- transfer level, RTL)、逻辑层、电路层、版图层, 才 能得到一个芯片. 如果上一层次的设计没有被充 分确认完成就进行下一层次的设计, 将带来非常 高的设计风险。

（5）芯片设计有明显的前后端分界，前后端设 计需用到不同领域的知识, 前端设计更类似于软 件设计，后端更多涉及工艺参数等物理域问题.

基于上述比较, 本文认为，将敏捷方法应用于 芯片设计领域, 其本质与该领域一直在追求的提 高设计生产率这一目标是一致的.

但与软件开发相比, 微处理器设计中应用敏 捷设计方法的范围和方式也存在很大差异. 虽然 存在各种困难，敏捷的核心理念——在设计过程 中，尽量集中在少的特征或功能上，基于不完整的 设计, 尽可能地深人至需要达到的深度, 把重点放 在设计者最担心的或者对其他部分有很大影响的 那一部分上——正逐步被集成电路设计领域接纳, 并已有较多的实践. 例如，即便设计还未达到 50\% 的程度, 也能考察时钟划分、功耗分布和可测性设 计等特性 ${ }^{\mathbb{1}}$.

除设计理念外，软件敏捷设计领域的各种技 术方法在芯片设计领域的应用也能有效支撑微处 理器敏捷设计, 例如测试驱动开发、构建跨职能团 队、结对编程等. 另一应用方法是将敏捷设计应用 于模拟验证与现场可编程逻辑门阵列 (field programmable gate array, FPGA)原型验证之间, 通过 敏捷方法和各种模拟器快速设计迭代, 一旦设计 稳定，立即切换到 FPGA 原型验证.

总之，相比把整个敏捷开发融人现有的开发 流程中，将敏捷设计方法分解开来，然后按实际情 况放人微处理器设计方法论和设计流程中, 会更 易于被设计人员接受.

\section{2 微处理器敏捷设计代表性工作}

敏捷的核心思想是提高微处理器设计的生产 率，而达成提高生产率的方法与手段是多样的. 在 微处理器设计领域, 对敏捷设计方法还没有明确 的定义, 各研究团队对敏捷的认识及达成方式的 探索各不相同. 最早意识到需要敏捷设计的是斯 坦福大学 VLSI 研究组 ${ }^{[1,7]}$, 几乎在相同的时间, 加 
州大学伯克利分校体系结构研究组围绕 RISC-V 也 开展了相关的研究 ${ }^{[8]}$. 这也是目前微处理器敏捷设 计研究的典型代表. 从 2012 年至今, 陆续还有康 奈尔大学计算机系统实验室 ${ }^{[9]}$ 等研究团队开展了 相关研究.

现有工作聚焦于将敏捷设计方法应用于微处 理器设计. 首先, 因为微处理器是非常复杂的数字 系统，在这类系统设计方法上的突破将带动在其 他数字系统上的应用. 其次, 相比其他数字系统, 微处理器设计更需要在上层设计或者设计早期, 规划整个系统的架构和设计，更早预见设计的合 理性，这契合了敏捷设计思想. 因此，目前的工作 主要聚焦于设计前端建模方法和微处理器生成方 法，以应对微处理器设计早期的时序更改、控制流 变化、系统异常处理等需求变更.

\section{1 斯坦福大学的工作}

斯坦福大学 VLSI 研究组首次提出了微处理器 生成器的概念, 以此来提升微处理器的设计生产 率，并设计了配套的原型工具 Genesis $2^{[1,7]}$. 其核 心理念是即便不可能为每个应用设计专用芯片, 但可以重用某个应用专用芯片的设计要素于新芯 片的设计

Genesis2 体系结构如图 1 所示, 进行微处理器 设计建模时, 主要发挥了 Verilog 和 Perl 语言的能 力, 将领域特定的设计知识和芯片设计折衷整合 为各种微处理器模板. 从这个角度看, Genesis2 也 是一个领域特定的微处理器生成器. 设计人员以 模板库为基础，编程调用模板库部件、配置各种参 数来构建各种芯片设计. Genesis2 将对设计者编写 的描述进行编译执行，在运行时生成具体的微处 理器设计描述. 这种方法也可看做硬件构建语言 (hardware construction language, HCL)的雉形, 其 显著特征是通过运行程序生成硬件设计. 基于 Genesis2, 斯坦福大学 VLSI 研究组实现了 $\mathrm{FPU}^{[10]}$, SSM 多核微处理器芯片 ${ }^{[11]}$ 生成器, 并基于该生成 器对芯片设计空间进行了探索.

Genesis2 的高层建模语言仍基于 Verilog (SystemVerilog)和 Perl，无法利用面向对象等建模 方法对敏捷设计的支持, 本质上仍是面向过程的 描述方法，存在较多不利于设计快速迭代的弱点. 因此, 斯坦福大学 AHA 敏捷硬件中心从 2017 年开

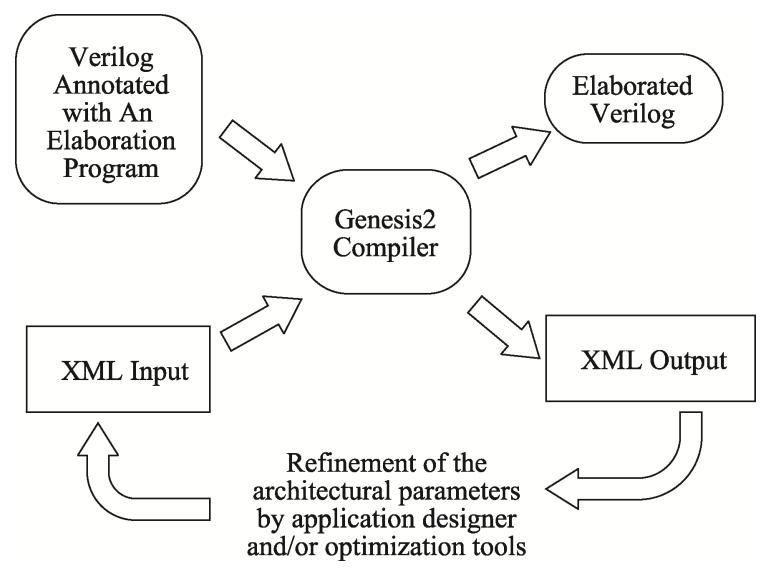

图 1 Genesis2 体系结构 ${ }^{[7]}$

始, 在 Genesis2 基础上，提出了一种新的设计方法 来实现微处理器敏捷设计. 该方法利用领域特定 语言(domain specific language, DSL)对现有方法进 行改进，并针对不同的设计模型设计了不同的 DSL. 在该设计方法中，底层基于粗粒度可重构体 系结构 (coarse grain reconfigurable architecture, CGRA $)^{[12]}$, 敏捷设计的目标是能快速将高层设计 映射到 CGRA 上, 进行快速设计探索、快速设计迭 代. 为了简化问题, AHA 针对 CGRA 不同的单元部 件设计了不同的 DSL, 针对 CGRA 中的处理单元 设计了 PEak 语言 ${ }^{\circledR}$, 针对内存设计了 Lake 语言 ${ }^{\circledR}$, 针对互联设计了 Canal 语言 ${ }^{3}$, 这些语言都是内嵌 于 Python ${ }^{\oplus}$ 的 DSL.

应用该方法设计微处理器时, 使用 Magma 语 言 ${ }^{5}$ 将 PEak, Lake 和 Canal 描述的设计转换为 RTL 设计, 作为物理设计的起点. 为应对物理设计参数 的变化, 提出了多阶段物理设计生成法, 并设计实 现了 Gemstone 原型系统 ${ }^{(0)}$. 多阶段物理设计生成 的核心理念是根据物理设计需要, 在 RTL 设计上 通过逐遍扫描，添加寄存器分布、全局信号布线、 功耗、时序等物理特性, 以便快速生成物理设计, 或简化布局布线等环节。

运用该方法, AHA 在 2 年内成功生产了 2 款 芯片 ${ }^{[5]}$.

\section{2 加州大学伯克利分校的工作}

该校体系结构实验室在 2016 年首次明确提出 了微处理器敏捷设计的概念 ${ }^{[4]}$, 仿照 Agile Manifesto 给出了 4 条敏捷硬件宣言 (agile hardware manifesto,

\footnotetext{
(1) https://github.com/cdonovick/peak

(2) https://github.com/StanfordAHA/lake

(3) https://github.com/StanfordAHA/canal
}

(4) https://www.python.org

(5) https://github.com/phanrahan/magma

(6) https://github.com/StanfordAHA/gemstone 
$\mathrm{AHM}$ ), 并针对每一条宣言给出了该实验室实践中 的做法:

（1）不完备的、可制造的原型系统，而不是特 征完整的模型：这条宣言重点强调微处理器敏捷 设计模型与传统瀑布模型的区别. 非常显著的区 别是在芯片设计的有效性验证方面，瀑布模型在 高层设计过早地引人了整个设计的模型以验证这 个设计是否满足设计要求, 并逐层对设计需求进 行精化, 以产出最终的芯片. 而敏捷设计方法强调 在高层实现不完备需求的设计，而使不完备设计 尽早产出时钟精确的 RTL 完整设计, 尽早进行时 序、面积和功耗等设计属性的评估，及早发现不可 满足的设计需求. 同时, 尽早利用 FPGA 运行实际 软件验证设计功能.

（2）灵活协作的团队，而不是刚性不变的开发 队伍: 瀑布模型中, 强调设计人员专精某个层次设 计所需的技术, 如前端的体系结构建模与设计探 索、RTL 建模，后端的物理设计与验证等，有时甚 至需将后端设计外包. 设计过程中，大量的时间用 于各层次设计师之间的交流. 而敏捷设计模型中, 强调的是一个小团队中，每个成员都知晓从前端 到后端各层次的设计与验证技术，每个这样的小 团队就能完成整个设计从前端到后端的设计流程.

（3）改进工具和生成器，而不是改进单个设计 实例: 利用嵌人在高级程序设计语言中的领域特 定 DSL 进行设计建模, 同时设计微处理器生成器, 在设计重用的基础上，更多地强调和实现设计流 程及工具的重用.

(4) 对变化响应, 而不是遵循某个计划: 为应 对设计需求的变更，每种设计成型的芯片，都将是 下一次迭代的起点. 此时, 将设计需求视为设计特 征, 尽早实现那些看起来不太会变化的设计特征, 而在此基础上, 不断迭代以加人新的设计特征. 倾 向于从一个小的核心设计出发, 不断扩展设计特 征和功能, 最终完成包含完整设计特征和功能微 处理器的设计. 强调前一代设计反馈对后一代设 计的帮助.

该方法在高层设计层面，通过提高描述层次， 利用面向对象设计等技术，用更精简的方法描述 RTL 设计. 这种设计描述语言是内嵌于 Scala ${ }^{\circledR}$ 语言 的 DSL, 称为 $\mathrm{Chisel}^{[8]}$, 目前已更新到 $\mathrm{Chisel}^{2}$. Chisel 本质上是一种 HCL, 即通过运行 Chisel 程序
构建硬件实现. Chisel 内建了各种数据类型以支持 在 RTL 层进行硬件建模, 通过设计模式、函数式 编程等技术，方便地表达设计、集成各种重用模块. Chisel 可以为设计生成高效的周期精确的 $\mathrm{C}++$ 模 拟模型, 或者使用标准工具生成适用于 FPGA 仿真 或 ASIC 综合的 Verilog 描述. 在验证方面, Chisel 带来的优势是编写 Testbench 与设计描述所用的语 法语义在同一个层次, 利用 Scala 提供的单元测试 等支持，加速设计验证.

该方法的另一个特点是快速集成特征，如图 2 所示, 多团队可以并行启动多个设计, 针对不同设 计特征进行深度探索，尽早发现哪些特征不合适. 而传统的开发模型, 要等所有的特征都准备好后, 才开始从前端到后端逐步设计.

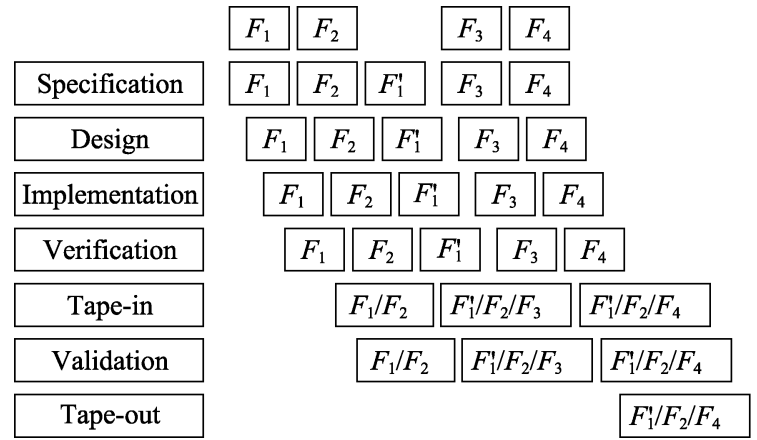

图 2 多特征并行探索与集成 ${ }^{[4]}$

加州大学伯克利分校体系结构实验室设计了 与微处理器敏捷设计方法相配套的一系列前端 EDA 工具 ${ }^{[13]}$, 包括 Chisel 语言、FIRRTL 中间格式 ${ }^{[14]}$, Rocket 芯片生成器 ${ }^{[15]}, \mathrm{BAG}$ 模拟电路生成器 ${ }^{[16]}$ 等, 围绕 RISC-V 实践了所提出的设计方法，在 5 年内 (2011-2015 年), 成功地进行了 12 次流片.

\section{3 其他工作}

英伟达公司(NVIDIA Corporation)、康奈尔大 学、哈佛大学和麻省理工学院联合开展了基于模块 化提升片上系统(system-on-a-chip, SoC)设计效率 的研究实践, 提出了一种面向“通用微处理器+机 器学习增强电路”片上系统的高效集成电路开发流 程—OOHLS $\mathrm{O}^{[17]}$, 如图 3 所示. 该流程包括高级 综合工具、可综合的 SystemC/C++面向对象组件库 MatchLib, 以及基于细粒度全局异步本地同步 (globally asynchronous locally synchronous, GALS) 时钟的模块化 VLSI 物理设计方法. MatchLib 为一

\footnotetext{
(1) https://www.scala-lang.org

(2) https://github.com/freechipsproject/chisel3
} 
个可综合 SystemC/C++面向对象组件库，主要用于 支持高级综合，而高级综合可视为比体系结构层 次稍低的一种生成器. MatchLib 包括 3 个层次的模 板电路, 分别为 $\mathrm{C}++$ 函数、 $\mathrm{C}++$ 类和 SystemC 模块. $\mathrm{C}++$ 函数是无时序的数据通路, $\mathrm{C}++$ 类是包含状态 和不定时方法或函数的对象，而 SystemC 模块用 于设计具有反馈或时钟行为的硬件单元.

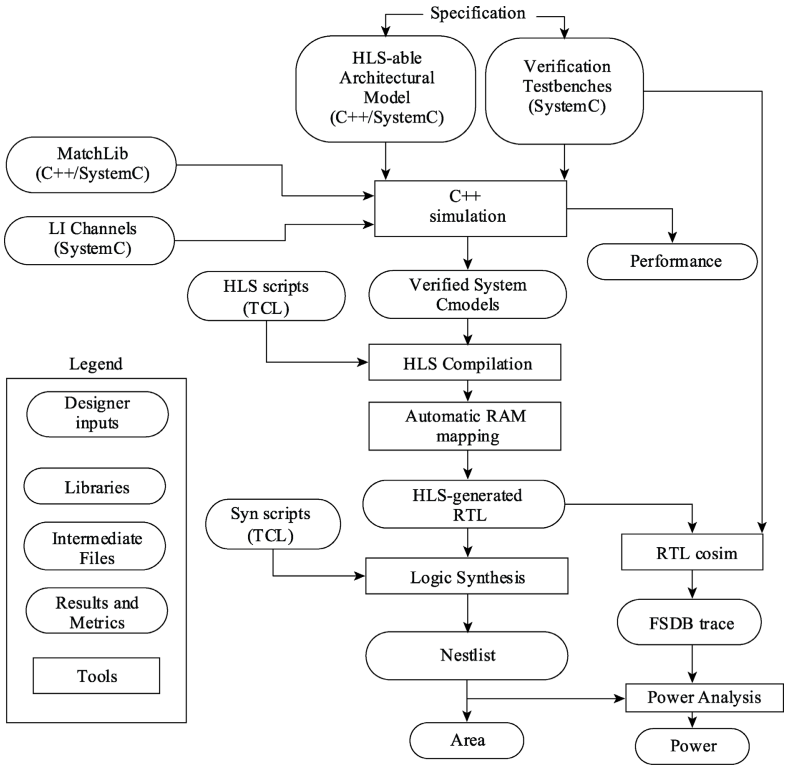

图 3 OOHLS 设计流程 ${ }^{[17]}$

OOHLS 流程中, 前端设计通过为常用部件自 行编写可综合 SystemC/C++组件库, 来弥补现有商 品化高级综合工具的不足; 后端设计仍采用传统 的设计流程和 EDA 工具. 因此, 可将该流程视为 是现有 EDA 工具的集成以支持新的设计流程，对 敏捷硬件设计方法学的探究较少.

该流程在采用 $16 \mathrm{~nm}$ FinFET 工艺，含 8700 万 晶体管的机器学习与计算机视觉 $\mathrm{SoC}$ 原型芯片上 得到了成功应用.

为了不断提高设计效率, 英飞凌公司在很大 程度上依赖于内部自动化框架 ${ }^{[18]}$, 该框架(如图 4 所示)基于内嵌于 Python 的硬件特定领域语言来描 述特定的设计需求. 为了能够处理多种映射目标, 该自动化框架遵循对象管理组织 (object management group, $\mathrm{OMG}$ )的模型驱动架构 (model driven architecture, MDA)规约，并集成了 DSL、元模型、 验证、代码生成等技术. 该框架本质上实现了模型 驱动设计方法 ${ }^{[19]}$, 即将设计规约与设计实现分离,

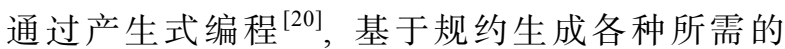
RTL 设计. 该自动化框架具有 2 个主要特点：(1) 统一定义的元模型形式化符号, 以此来规范覆盖
整个芯片设计空间的不同的硬件 DSL. (2) 为硬件 DSL 生成的中间模型定义了形式化语义, 以支持 对硬件 DSL 生成的硬件描述进行验证.

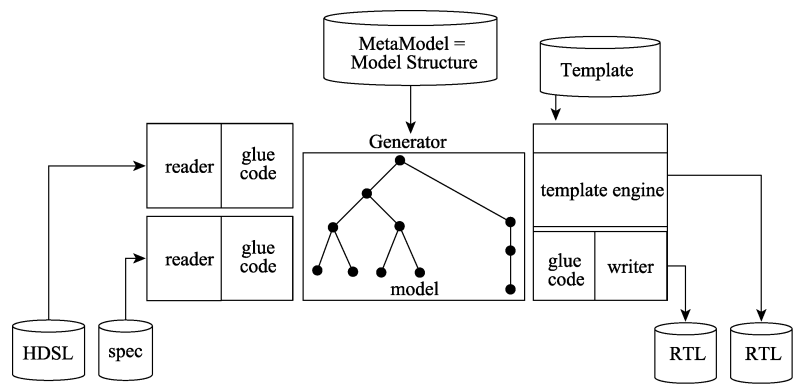

图 4 基于模型的设计流程 ${ }^{[18]}$

国防科技大学将面向特征 ${ }^{[21]}$ 的软件开发方 法引人 $\mathrm{SoC}$ 高层设计, 以 SystemC 为高层建模方 法, 提出了 SystemC 模型产品线 (SystemC product line, SCPL ${ }^{[22-24]}$ 概念, 研究了支持 SCPL 的开 发方法、组合安全性和形式化功能验证等问题, 在 OpenRisc 1000 项目和 MJPEG 解码芯片项目上的 应用，验证了该方法在支持 $\mathrm{SoC}$ 高层快速设计上 的有效性 ${ }^{[25]}$.

中国科学院计算技术研究所在基于 Chisel 和 RISC-V 的微处理器敏捷设计方面开展了较深人和 广泛的应用研究, 总结了基于 RISC-V 开源芯片和 Chisel 工具集的敏捷设计方法 ${ }^{[26]}$, 并基于该方法 和相应工具集, 开展了标签化 RISC-V 的设计与实 现研究 ${ }^{[27]}$, 验证了微处理器敏捷设计方法对设计 效率的提升作用. 北京大学在开源 EDA 方面有较 为深人的研究 ${ }^{[28]}$, 业界认为, 支撑微处理器敏捷 设计的有效途径之一是利用开源 EDA 工具集.

\section{3 敏捷设计方法关键使能技术}

将敏捷设计方法应用到微处理器设计领域, 必将对设计方法及支撑设计方法的 EDA 技术带来 深刻影响. 本文将那些由于这些技术而使得微处 理器敏捷设计成为可能并取得成功的新技术称为 使能技术，其中的关键使能技术有以下几种.

\section{1 前端建模技术}

目前的前端建模仍以 RTL 设计为建模目标, 主要解决 Verilog(SystemVerilog) 等硬件描述语言 对设计抽象、关注度分离、模块化等鼓励代码重用 机制支持弱的问题. 主流技术是依托某种高级程 序设计语言(如 Scala，Python)设计 DSL，通过定义 支持 RTL 建模的端口、寄存器、连线、逻辑单元 
类等语言机制，扩展现有语言的硬件描述能力, 并 在运行程序时, 自动生成 Verilog 或其他格式的 RTL 电路描述, 这种 DSL 又称为硬件构建语言, 其所依托的语言称为宿主语言.

首先，这种 DSL 的优点是具有：

(1) 充分发挥高级程序设计语言灵活方便的 表达能力;

(2) 充分发挥高级程序设计语言在面向对象 设计与描述、参数化、模块化等方面的支持能力;

(3) 充分利用软件开发方法中已经较为成功 的设计重用、设计模式等应对用户需求变化的设计 技术的能力.

通过上述能力，实现高层设计的快速变更、快 速迭代.

其次，这种 DSL 在语义层面统一了可综合与 模拟语义, 克服了 Verilog 等语言在设计和模拟语 义上的不一致问题.

第三, DSL 宿主语言表达能力为可重用硬件库 的开发奠定了坚实的基础.

从 20 世纪 90 年代开始，陆续出现了基于某种 高级程序设计语言设计 DSL 进行 RTL 设计建模的

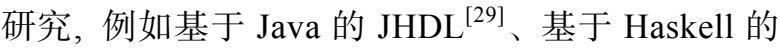
$\mathrm{C} \mathrm{aSH}^{[30]}$ 、基于 $\mathrm{ML}$ 语言的 $\mathrm{HML}^{[31]}$ ，以及以中间语 言形式出现的 DIL $^{[32]}$. 这些语言在设计上将 HDL 抽象机制与无硬件模型且语义完全不同的高级语 言相结合，在进行设计建模时，显得较为笨重.

目前主流 DSL 大多基于 Scala 或 Python 构建, 较为典型的有 Chisel, PyRTL 等. Chisel ${ }^{[8]}$ 是一个基 于 Scala 的硬件构建语言. Chisel 内建了各种数据 类型以支持在高层次进行硬件建模，通过设计模 式技术，在各种项目中方便地集成各种重用模块. Chisel 可以为设计生成高效的周期精确的 $\mathrm{C}++$ 模 拟模型，或者使用标准工具生成适用于 FPGA 仿真 或 ASIC 综合的 Verilog 描述。该语言已在 OpenRISC 和 RISC-V 等项目上进行了实践验证. 但该语言的工具链仍不够完整.

PyRTL ${ }^{[33]}$ 在 Python 的基础上提供了一组 RTL 建模的原语，以支持精准的设计描述，利用设计模 式技术提高设计建模的效率, 要求所有逻辑都是 同步和可综合的，也是一种基于执行的设计构造 语言. 该语言主要用于教学与科研, 有一个适合于 初学者快速人门的工具链. PyRTL 的目标是简单、 易用、可扩展，支持 RTL 设计描述、模拟、测试
与跟踪等. 目前, 支持与 Xilinx PYNQ 的集成, 便 于 $\mathrm{SoC}$ 的快速原型开发. PyRTL 在设计时对标了 Chisel，其实现的语言机制与 Chisel 机制非常相似. 基于 Python 语言定制的代表性硬件描述 DSL 还有 $\mathrm{MyHDL}^{[34]}$, PyMTL ${ }^{[35]}$, SysPy ${ }^{[36]}$ 和 $\mathrm{PHDL}^{[37]}$ 等，这些语言针对不同的应用目标，利用 Python 提供的语言机制，在不同方面支持高层硬件描述、 模拟与仿真, 以及硬件综合.

需要注意的是, $\mathrm{HCL}$ 本身不提供任何新的硬 件抽象, 仍是 RTL 建模, 但是宿主语言特征能使 设计变得更加参数化和模块化.

\section{2 中间表示及其处理}

微处理器敏捷设计方法的前端建模, 大量借 鉴了编译技术，引人类似于 $\mathrm{LLVM}^{[38]}$ 的中间格式. 在将 HCL 描述编译为中间格式后, 可在此基础上 快速地评估各种后端设计参数对设计的影响, 或 基于中间格式发展出各种验证技术，如符号模拟、 等价性检查、模型检验等。

中间格式对敏捷设计的支持表现在对“ASIC 设计需要根据工艺参数对 RTL 设计进行定制” 的支 持上. 传统方法是通过编写一组自定义脚本对 RTL 设计进行特殊的可编程的修改. 但是, 这些脚 本既不可重用, 又不鲁棒, 也不可组合. 而基于中 间格式表示, 可将对 RTL 的定制编写为函数, 利 用 HCL 宿主语言的高阶函数机制, 将定制函数作 为参数传人, 递归地将定制动作作用到设计的各 部分. 由于这种定制被封装于函数内，且可作为其 他函数的参数，使得定制与 RTL 设计是松耦合的， 极大地提高了定制的可重用性、可快速变更性. 这 种定制也可被替换为设计优化、设计化简、面积功 耗预估、到 FPGA 或 ASIC 的映射、对验证的支持 等. 代表性的中间格式有 FIRRTL ${ }^{[14]}$ 和 CoreIR ${ }^{\circledR}$.

FIRRTL 的设计原则是清晰(语义直接清晰)、 简单(中间格式元素尽量少)、丰富(能完全满足设计 意图的表达)，与此对应，FIRRTL 中定义了 circuit, module, port, statement, expression 和 type 等节点元 素, 如图 5 所示. 某个设计的 FIRRTL 表示在内存 中以抽象语法树(abstract syntax tree, AST)形式呈 现，基于这种表示，利用递归遍历 AST，同时将各 种转换直接映射到节点上, 实现多种设计转换, FIRRTL 中间格式的转换处理如图 6 所示.

CoreIR中间格式主要为微处理器生成器而设计, 是一种类似于 LLVM 的独立于前后端工具的格式. 


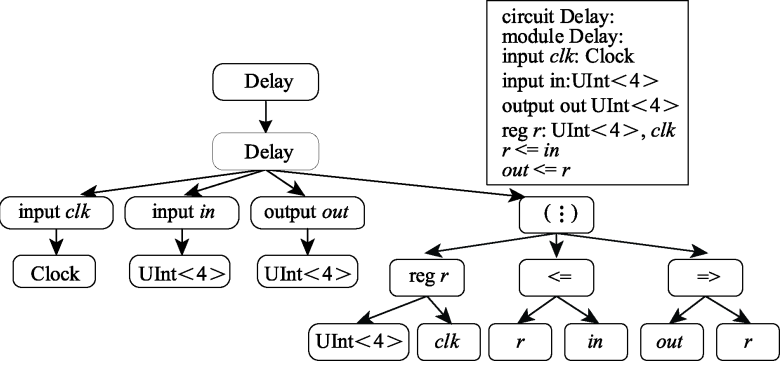

图 5 定义的元素及 AST 表示示例 ${ }^{[14]}$

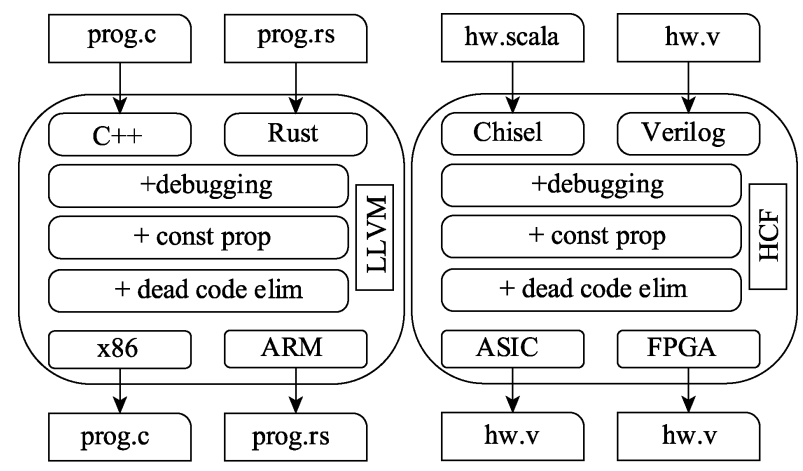

图 6 FIRRTL 中间格式的处理 ${ }^{[14]}$

它设计了完备的硬件元语，大量采用位向量数据类 型, 提供较多的类似于 RISC-V 指令集的元语与扩 展. 这些机制使 CoreIR 表示能很方便地集成进形式 化验证工具链中, 也能很好地支持微处理器生成器. 在斯坦福大学 AHA 敏捷硬件项目提出的微处理器 敏捷设计流程中, 以 CoreIR 为核心, 串联起各种

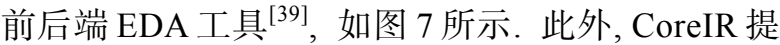
供了各种可扩展接口, 用于创建各类优化、分析和 转换工具. 这些接口也可用于将工艺参数映射到 CoreIR 设计元语，进行时序、功耗等分析，并直接 生成物理设计.

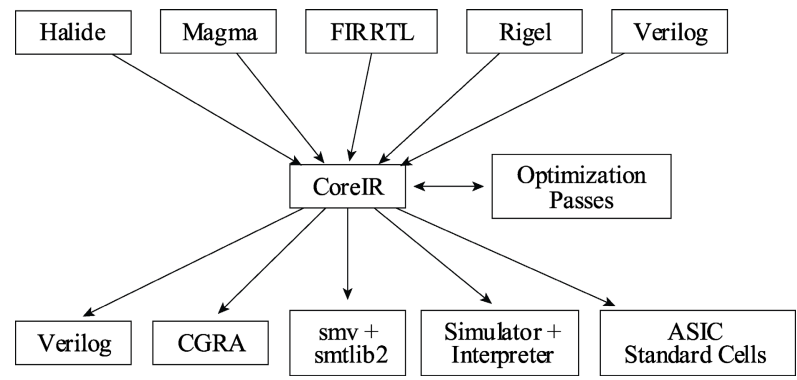

图 7 以 CoreIR 为核心的敏捷设计流程 ${ }^{[39]}$

\section{3 微处理器生成技术}

微处理器生成器的核心思想是避免设计单个 芯片实例, 而是通过设计生成器, 配置参数, 自动
生成满足需求的微处理器 ${ }^{[1]}$, 其关键技术是微处理 器特征参数的建模、模板电路、模板体系结构的 设计, 这些技术涉及的内容常常与应用领域密切 相关.

Genesis $2^{[11]}$ 是一款典型的领域特定微处理器 生成器. 为了利用 SystemVerilog 的验证能力, Genesis2 采用 SystemVerilog 作为描述语言, 构建了大 量的模板电路, 以及领域特定的体系结构模板. 利 用 Perl 作为脚本语言来配置体系结构参数, 实例 化模板电路, 组合出满足要求的微处理器体系结 构设计. 严格意义上来说, Genesis2 是一个构造各 种芯片生成器的工具, 即元生成器.

图 8 的程序展示了 Genesis2 的核心技术理念, 其中“//;”开始的语句表示该行语句为 Perl 语句, 将 按照 Perl 语言的语义进行解析执行. 未以“//;”开始 的语句为 Verilog 语句，其中以“\$”开始的变量可视 为设计中的“空洞”, 这些空洞也将按 Perl 语义解释 并执行.

$/ /$ my \$width $=5$;

assign some wire['\$width-1':0] = some other wire['\$width+9':10]; //; foreach my $\$ i d x(0,1,2,3)\{$ assign wire ' $\$ i d x '=$ wire_' $(\$ i d x+1) \% 4$ '; $\| ;\}$

图 8 Genesis2 示例程序

当用 Genesis2 执行该程序时，将执行其中的 Perl 语句，填充 Verilog 语句中的空洞，生成具体的 Verilog 描述. 图 8 的示例程序经 Genesis2 执行后 的输出如图 9 所示.

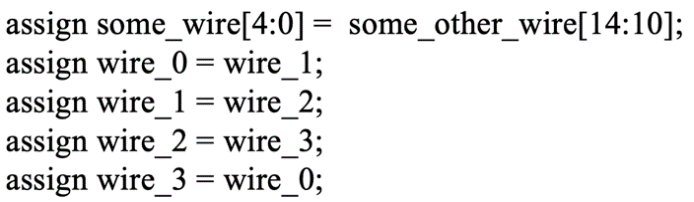

图 9 Genesis2 生成程序示例

基于这样的理念和设计, Genesis2 能根据模板 快速地生成微处理器部件及微处理器整体设计.

除了生成设计, Genesis2 还将同时生成附带的 验证支持，包括生成 Testbench、设计断言、测试激 励以及参考模型, 以此加速设计验证. 但验证仍以 模拟方法为主.

在已有实践基础上，AHA 敏捷硬件项目对 Genesis2 方法进行了改进. 首先提出了新的 CGRA 体系结构 Garnet ${ }^{\mathbb{D}}$, 相比第 1 代体系结构, 增强了 
处理单元能力、提供了 BFloat 和超越计算功能, 以 及支持神经网络的全局缓冲区、用于低功耗的可配 置电源管理等, 以实现更快速的可重构. 以 Garnet 为生成目标, 利用 Magma 语言设计可重用 CGRA 部件, 如处理单元、存储单元和互连网络等. Magma 也是一种 HCL, 在执行时生成所描述的设 计单元. 具体的生成由 Gemstone 完成, Gemstone 含有融合上述各种设计单元所需的逻辑, 并提供 了定制设计的机制，通过定制支持机制可对设计 进行多阶段式转换, 以与其他设计阶段, 如物理 设计、验证等相匹配. 多阶段转换是在 RTL 设计 上通过逐遍扫描，添加其他设计阶段操作所需的 特性, 如寄存器分布、全局信号布线、功耗、时 序等物理特性. 该工作仍处于起始阶段, 公开发 表于文献[5].

Rocket Chip ${ }^{[15]}$ 是一款开源 $\mathrm{SoC}$ 设计生成器, 可生成可综合的 RTL 设计. Rocket Chip 需要各种 设计模板的支持, 这些模板包括微处理器内核 (core)、高速缓存(cache)和复杂互连等. 在设计时, 利用 Chisel 硬件构造语言, 将所需的电路模板(参 数化)组合进设计中，通过运行描述设计的 Chisel 程序, 自动地将集成的电路模板组合成一个完整 SoC. Rocket Chip 生成器使用开放式 RISC-V 指令 集的通用处理器内核, 并提供有序执行内核生成 器 (Rocket) 和无序执行内核生成器 (BOOM $)^{[40]}$. Rocket Chip 还支持以指令集扩展、协处理器或完 全独立的新型内核的形式，在 RICS-V 上集成定制 加速器, 以提高微处理器设计效率. 本质上, Rocket Chip 生成器是一种强大而丰富的库资源, 是对 Chisel 生态提升设计生产率的有力补充.

综上, 目前的微处理器生成器基本是基于特 定目标体系结构的，如斯坦福大学的 CGRA、加州 大学伯克利分校的 RISC-V，针对通用或可变体系 结构的微处理器生成器的研究还非常少.

\section{4 后端设计敏捷方法}

为敏捷化后端设计流程，主要技术思路是在 整个后端设计过程中, 尽可能减少人工干预，定期 为设计师提供芯片时钟周期、面积和功耗等性能反 馈，使得在后端设计早期能预测更多物理设计问 题, 减少后期设计返工.

在使用硬件描述生成电路版图文件 GDSII (geometry data standard II)的过程中, 需要多次设 计迭代, 而每次迭代可能需要数小时才能完成, 对于大型设计, 一般需要数周或数月的时间. 为避 免长时间迭代影响设计效率，文献[4]在 RISC-V 设
计上的实践表明，可以主要关注较小的设计，更 快地生成 GDSII. 此外, 硬件生成器的可参数化、 标签化特性，在设计规模扩大时，可以减少所需 的工作量. 而对于每个设计, 需要确保其通过静 态时序分析, 功能上等同于 RTL 模型. 最后, 当 前芯片设计完成后, 会对电路版图进行打包并测 试，将当前单个芯片设计信息整合反馈到芯片系 列中, 为下一代芯片设计提供经验数据.

\section{5 设计验证方法}

目前, 微处理器敏捷设计方法研究更多关注 设计层面的技术, 对验证技术方面关注较少, 基本 可分为形式化验证和模拟验证 2 类.

形式化验证方面, 主要有针对敏捷设计方法的 $\mathrm{CoSA}^{[41]}$ 系统. 该系统基于 CoreIR 中间格式, 将所 有对设计的分析问题转换为符号模型检验 ${ }^{[42]}$ 问题, 基于限界模型检验技术 ${ }^{[43]}$ 与 SMT 求解技术 ${ }^{[44]}$, 分 别用 K-Induction ${ }^{[45]} /$ Interpolation $^{[46]}$ 与 K-Liveness ${ }^{[47]}$ 技术处理安全和活性性质的验证，通过自动机乘 积进行等价性检查. CoSA 系统作为验证支撑，已 被集成进斯坦福大学 AHA 敏捷硬件项目中. 此外, 针对敏捷设计方法对多特征设计的并行探索，文 献[48]提出了一种设计族验证优化技术, 可指数级 地减少待验证的设计数量, 在 OpenRisc 1000 项目 和 MJPEG 解码芯片项目上得到了成功应用.

由于前端描述方法为内嵌于 Scala 或 Python 语言的 DSL, 导致模拟效率下降, 因此, 目前模拟 验证方面的研究主要集中在模拟效率的提升. 通 常有 2 种改进方法: (1) 对模拟引擎进行优化, 以 提高模拟效率。代表性工作有康奈尔大学的 Mamba 模拟框架 ${ }^{[49]}$, Mamba 对 Python 解释执行机 制进行优化, 提出了针对 JIT 编译的模拟代码生成 技术, 以及针对模拟的 JIT 数据类型和大循环缓冲 技术, 改进后模拟性能与商用模拟器性能相当. (2) 利用 FPGA 或硬件云平台, 提升模拟效率 ${ }^{[50-51]}$. 代 表性工作有加州大学伯克利分校的 RFUZZ 系统 ${ }^{[52]}$, 该系统将设计插桩后导人 FPGA, 提升模糊测试的 性能.

\section{4 微处理器敏捷设计方法的研究方向}

综上可知, 微处理器敏捷设计方法强调硬件 设计的快速开发迭代. 主要方法和技术有:

(1) 通过提高描述层次，利用面向对象等技术 用更精简的方法描述硬件设计;

(2) 通过各种库支持设计重用, 自动生成可综 
合的设计描述;

(3) 利用与设计层次相同层次的测试, 加速设 计验证进程;

（4）通过快速集成设计特征，同步进行多个版 本的设计

这些方法与技术极大地提高了设计生产率. 通过已有的研究实践表明, 在微处理器设计中采用 敏捷设计方法是可行的, 但仍需要在新设计方法的 理论、技术体系、应用方法等方面开展深人研究.

此处列出本文认为可真正实现“高层一RTLGDSII”端到端敏捷设计的研究方向:

(1) 更高抽象级别的建模.例如虚拟机级别, 即将硬件建模为用目标机器指令集构建的程序, 通过现有 RTL 建模或新技术的支持，实现端到端 映射，将虚拟机建模直接映射到硬件实现的微处 理器生成技术.

（2）建立基于高级程序设计语言的语言族. 针 对微处理器各部件不同特性, 设计不同的建模方 法, 研究如何充分发挥高级程序设计语言编译与 多阶段优化、模板、协程等基础设施机制，实现各 种建模 DSL 的机制统一.

(3) 采纳更多软件开发技术. 在应对用户需求 变化方面, 软件开发领域涌现了大量的方法, 如面 向对象、设计模式、面向方面、面向特征等设计技 术，如何将这些已经证明有效的方法应用到硬件 设计，提升设计效率，也将是研究方向之一.

（4）基于机器学习的无人干预后端预测一反 馈快速迭代. 通过机器学习方法准确预测后端下 游各阶段的输出结果, 并将预测后的结果, 反馈指 导设计优化，真正实现从 “RTL-to-GDSII”的端 到端设计。

（5）端到端验证优化技术. 验证是设计正确性 和有效性的重要保障，当设计技术支持大跨度端 到端转换时，验证技术必须能与这种变化相匹配， 要求验证也要尽可能敏捷. 此外, 随着芯片设计的 “软”化，在软件验证领域有成功应用的静态分析、 产品线验证等技术, 也将在微处理器敏捷设计流 程中得到广泛应用.

(6) 前后端设计的无缝衔接技术. 目前的研究 聚焦于前端设计建模，以应对敏捷设计带来时序 更改、控制流变化、系统异常处理等需求变更，但 后端设计仍以现有设计流程和方法为主. 微处理 器具有功能部件的构成比较固定, 通过不同配置 的部件, 组合成系列设计. 这种构成较为固定的特 性有利于充分利用前后端历史设计数据，预测新
设计的时序、功耗、面积等物理特性. 这涉及前后 端设计信息表示方式整合、数据压缩和划分、设计 样本扩充，以及特征提取等技术，实现前后端“大 跨度”的工具和流程预测，以把上游设计的结果，快 速准确地指向到设计下游甚至设计末端预测.

\section{5 结 语}

本文对当前微处理器敏捷设计的研究现状进 行了综述, 包括微处理器敏捷设计的本质含义、研 究实践和应用效果等; 进而归纳并分析了目前支 持微处理器敏捷设计的关键使能技术; 最后给出 了潜在的研究方向, 希望对本领域的科研人员有 所帮助.

\section{参考文献(References):}

[1] Shacham O, Azizi O, Wachs M, et al. Rethinking digital design: why design must change[J]. IEEE Micro, 2010, 30(6): 9-24

[2] Hennessy J L, Patterson D A. A new golden age for computer architecture[J]. Communications of the ACM, 2019, 62(2): 48-60

[3] Salmon L. Circuit realization at faster timescales (CRAFT)[OL]. [2020-02-09]. https://eri-summit.darpa.mil/ docs/Salmon_Linton_CRAFT_Final.pdf

[4] Lee Y, Waterman A, Cook H, et al. An agile approach to building RISC-V microprocessors[J]. IEEE Micro, 2016, 36(2): 8-20

[5] Bahr R, Barrett C, Bhagdikar N, et.al. Creating an agile hardware flow[C] //Proceedings of Hot Chips. Los Alamitos: IEEE Computer Society Press, 2019: 1-29

[6] Fowler M, Highsmith J. The agile manifesto[OL]. [2020-02-09]. http://www.drdobbs.com/184414755

[7] Shacham O, Galal S, Sankaranarayanan S, et al. Avoiding game over: bringing design to the next level[C] //Proceedings of the 49th Annual Design Automation Conference. New York: ACM Press, 2012: 623-629

[8] Bachrach J, Vo H, Richards B, et al. Chisel: constructing hardware in a Scala embedded language[C] //Proceedings of the 49th Annual Design Automation Conference. New York: ACM Press, 2012: 1216-1225

[9] Jiang S N, Torng C, Batten C. An open-source Python-based hardware generation, simulation, and verification framework[C] //Proceedings of the 1st Workshop on Open-Source EDA Technology. Redmond: GitHub, 2018: Article No.13

[10] Galal S, Shacham O, Brunhaver II J S, et al. FPU generator for design space exploration[C] //Proceedings of the 21st IEEE Symposium on Computer Arithmetic. Los Alamitos: IEEE Computer Society Press, 2013: 25-34

[11] Shacham O. Chip multiprocessor generator: automatic generation of custom and heterogeneous compute platforms[D]. Palo Alto: Stanford University, 2011 
[12] Nayak A, Zhang K Y, Setaluri R, et al. A framework for adding low-overhead, fine-grained power domains to CGRAs[C] // Proceedings of the Design, Automation \& Test in Europe Conference \& Exhibition. Los Alamitos: IEEE Computer Society Press, 2020: 846-851

[13] Alon E, Asanović K, Bachrach J, et al. Open-source EDA tools and IP, a view from the trenches[C] //Proceedings of the 56th Annual Design Automation Conference. New York: ACM Press, 2019: 1-3

[14] Izraelevitz A, Koenig J, Li P, et al. Reusability is FIRRTL ground: hardware construction languages, compiler frameworks, and transformations[C] //Proceedings of the IEEE/ACM International Conference on Computer-Aided Design. Los Alamitos: IEEE Computer Society Press, 2017: 209-216

[15] Asanovic K, Avizienis R, Bachrach J, et al. The Rocket chip generator[R]. Berkeley: University of California, Berkeley. EECS Department, 2016

[16] Crossley J, Puggelli A, Le H P, et al. BAG: a designer-oriented integrated framework for the development of AMS circuit generators[C] //Proceedings of the IEEE/ACM International Conference on Computer-Aided Design. Los Alamitos: IEEE Computer Society Press, 2013: 74-81

[17] Khailany B, Khmer E, Venkatesan R, et al. A modular digital VLSI flow for high-productivity SoC design[C] //Proceedings of the 55th Annual Design Automation Conference. New York: ACM Press, 2018: Article No.72

[18] Devarajegowda K, Schreiner J, Findenig R, et al. Python based framework for HDSLs with an underlying formal semantics[C] //Proceedings of the 36th International Conference on Computer-Aided Design. Los Alamitos: IEEE Computer Society Press, 2017: 1019-1025

[19] Siegel J M. MDA guide revision 2.0[OL]. [2020-02-09]. https:/www.omg.org/cgi-bin/doc?ormsc/14-06-01.pdf

[20] Czarnecki K, Eisenecker U. Generative programming: methods, tools, and applications[M]. Beijing: China Electric Power Press, 2004(in Chinese)

(Czarnecki K, Eisenecker U. 产生式编程一方法、工具与应 用 $[\mathrm{M}]$. 梁海华, 译. 北京: 中国电力出版社, 2004)

[21] Prehofer C. Feature-oriented programming: a fresh look at objects[C] //Proceedings of European Conference on ObjectOriented Programming. Heidelberg: Springer, 1997: 419-443

[22] Ye J, Tan Q P, Li T. Separation of communication and computation in SystemC/TLM modeling: a feature-oriented approach[C] //Proceedings of the 12th International Symposium on Quality Electronic Design. Los Alamitos: IEEE Computer Society Press, 2011: 1-5

[23] Ye J, Tan Q P, Li T. Towards the development of a set of transaction level models-a feature-oriented approach[M] //System Specification and Design Languages. Heidelberg: Springer, 2012: 143-156

[24] Ye J, Tan Q P, Li T, et al. Feature-oriented refactoring proposal for transaction level models in SoCLib[C] //Proceedings of the Forum on Specification \& Design Languages. Los Alamitos: IEEE Computer Society Press, 2010: 1-6

[25] Ye Jun. Feature-oriented development and formal functional verification of SystemC model product line[D]. Changsha: National University of Defense Technology, 2011(in Chinese)
(叶俊. 面向特征的 SystemC 模型产品线的开发和形式化功 能验证技术研究[D]. 长沙: 国防科学技术大学, 2011)

[26] Wang Huizhe, Tang Dan, Yu Zihao, et al. Open-source chip, RISC-V and agile development[J]. Big Data Research, 2019, 5(4): 50-66(in Chinese)

(王诲喆，唐丹，余子濠，等. 开源芯片、RISC-V 与敏捷开发 [J]. 大数据, 2019, 5(4): 50-66)

[27] Yu Zihao, Liu Zhigang, Li Yiwei, et al. Practice of chip agile development: labeled RISC-V[J]. Journal of Computer Research and Development, 2019, 56(1): 35-48(in Chinese) (余子濠，刘志刚，李一苇，等. 芯片敏捷开发实践：标签化 RISC-V[J]. 计算机研究与发展, 2019, 56(1): 35-48)

[28] Wang Shuo, Zhang Jiaxi, Luo Guojie, et al. Open-source hardware and open-source EDA tools: accelerator for future chip design[J]. Frontier Science, 2018, 12(4): 56-60(in Chinese) (王硕, 章嘉叕, 罗国杰, 等. 开源硬件与开源 EDA 工具: 芯 片未来设计的加速器 $[J]$. 前沿科学, 2018, 12(4): 56-60)

[29] Bellows P, Hutchings B. JHDL - an HDL for reconfigurable systems[C] //Proceedings of the IEEE Symposium on FPGAs for Custom Computing Machines. Los Alamitos: IEEE Computer Society Press, 1998: 175-184

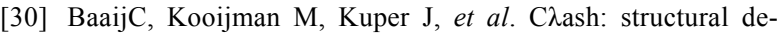
scriptions of synchronous hardware using Haskell[C] // Proceedings of the Euromicro Conference on Digital System Design. Los Alamitos: IEEE Computer Society Press, 2010, 1: 714-721

[31] Li Y B, Leeser M. HML - a novel hardware description language and its translation to VHDL[J]. IEEE Transactions on Very Large Scale Integration Systems, 2000, 8(1): 1-8

[32] Budiu M, Goldstein S C. Fast compilation for pipelined reconfigurable fabrics[C] //Proceedings of the 7th ACM/SIGDA International Symposium on Field Programmable Gate Arrays. New York: ACM Press, 1999: 195-205

[33] Clow J, Tzimpragos G, Dangwal D, et al. A pythonic approach for rapid hardware prototyping and instrumentation[C] // Proceedings of the 27th International Conference on Field Programmable Logic and Applications. Los Alamitos: IEEE Computer Society Press, 2017: 1-7

[34] Decaluwe J. MyHDL: a Python-based hardware description language[J]. Linux Journal, 2004, 2004(127): 84-89

[35] Lockhart D, Zibrat G, Batten C. PyMTL: a unified framework for vertically integrated computer architecture research[C] // Proceedings of the 47th Annual IEEE/ACM International Symposium on Microarchitecture. Los Alamitos: IEEE Computer Society Press, 2014: 280-292

[36] Logaras E, Manolakos E S. SysPy: using Python for processor-centric SoC design[C] //Proceedings of the 17th IEEE International Conference on Electronics, Circuits, and Systems. Los Alamitos: IEEE Computer Society Press, 2010: 762-765

[37] Mashtizadeh A. PHDL: a Python hardware design framework[D]. Cambridge: Massachusetts Institute of Technology, 2007

[38] Lattner C, Adve V. LLVM: a compilation framework for lifelong program analysis \& transformation[C] //Proceedings of the IEEE/ACM International Symposium on Code Generation and Optimization. Los Alamitos: IEEE Computer Society Press, 2004: 75 
[39] Daly R, Truong L, Hanrahn P. Invoking and linking generators from multiple hardware languages using CoreIR[C] // Proceedings of the 1st Workshop on Open-Source EDA Technology. Redmond: GitHub, 2018: 11.1-11.5

[40] Celio C, Patterson D A, Asanovic K. The Berkeley out-of-order machine (BOOM): an industry-competitive, synthesizable, parameterized RISC-V processor[R]. Berkeley: University of California, Berkeley. EECS Department, 2015

[41] Mattarei C, Mann M, Barrett C, et al. CoSA: integrated verification for agile hardware design[C] //Proceedings of the Formal Methods in Computer Aided Design. Los Alamitos: IEEE Computer Society Press, 2018: 1-5

[42] Clarke E M, Henzinger T A, Veith H, et al. Handbook of model checking[M]. Heidelberg: Springer, 2018

[43] Biere A, Cimatti A, Clarke E M, et al. Bounded model checking[J]. Advances in Computers, 2003, 58: 117-148

[44] BBarrett C, Tinelli C. Satisfiability modulo theories[M] // Handbook of Satisfiability. Amsterdam: IOS Press, 2009, 185: 825-885

[45] Sheeran M, Singh S, Stålmarck G. Checking safety properties using induction and a SAT-solver[C] //Proceedings of the 3rd International Conference on Formal Methods in Computer-Aided Design. Heidelberg: Springer, 2000: 127-144

[46] McMillan K L. Interpolation and SAT-based model checking[C] //Proceedings of International Conference on Computer
Aided Verification. Heidelberg: Springer, 2003: 1-13

[47] Claessen K, Sörensson N. A liveness checking algorithm that counts[C] //Proceedings of the Formal Methods in Computer-Aided Design. Los Alamitos: IEEE Computer Society Press, 2012: 52-59

[48] Li T, Ye J, Tan Q P. Towards functional verifying a family of SystemC TLMs[J]. Frontiers of Computer Science, 2020, 14(1): 53-66

[49] Jiang S N, Ilbeyi B, Batten C. Mamba: closing the performance gap in productive hardware development frameworks[C] // Proceedings of the 55th Annual Design Automation Conference. New York: ACM Press, 2018: Article No.60

[50] Kim D, Celio C, Karandikar S, et al. Debugging RISC-V processors with FPGA-accelerated RTL simulation in the FPGA cloud[C] //Proceedings of the 2nd Workshop on Computer Architecture Research with RISC-V, at the 45th International Symposium on Computer Architecture. New York: ACM Press, 2018: $1-7$

[51] Karandikar S, Mao H, Kim D, et al. FireSim: FPGA-accelerated cycle-exact scale-out system simulation in the public cloud[J]. IEEE Micro, 2019, 39(3): 56-65

[52] Laeufer K, Koenig J, Kim D, et al. RFUZZ: coverage-directed fuzz testing of RTL on FPGAs[C] //Proceedings of the International Conference on Computer-Aided Design. New York: ACM Press, 2018: Article No.28 